\title{
Chopper-Stabilized Instrumentation Amplifier with Automatic Frequency Tuning Loop
}

\author{
Chen-Mao Wu ${ }^{1}$, Hsiao-Chin Chen ${ }^{2}{ }^{10}$, Ming-Yu Yen ${ }^{2, *}$ and San-Ching Yang ${ }^{3}$ \\ 1 Asmedia Technology Inc., New Taipei 231, Taiwan; alexwuee2b@gmail.com \\ 2 Department of Electrical Engineering, National Taiwan University of Science and Technology, \\ Taipei 10607, Taiwan; hcchen@mail.ntust.edu.tw \\ 3 Inventec Corporation, Taipei 11107, Taiwan; m10207414@mail.ntust.edu.tw \\ * Correspondence: d10207401@mail.ntust.edu.tw; Tel.: +886-2-2733-3141 (ext. 7399)
}

Received: 14 May 2018; Accepted: 29 May 2018; Published: 8 June 2018

check for updates

\begin{abstract}
A variable-gain chopper-stabilized instrumentation amplifier (chopper IA), which employs a low pass filter (LPF) to attenuate the up-converted noise at the chopping frequency, is presented. The circuit is designed and fabricated with Taiwan Semiconductor Manufacturing Company (TSMC) (Hsinchu, Taiwan) $0.18 \mu \mathrm{m}$ complementary metal-oxide-semiconductor (CMOS) technology. Consuming $1.1 \mathrm{~mW}$ from a $1.2 \mathrm{~V}$ supply voltage, the chopper IA achieves a variable gain of 20.7-48.5 dB, with a minimum bandwidth of $6.7 \mathrm{kHz}$ and a common-mode rejection ratio (CMRR) of $95 \mathrm{~dB}$ below $10 \mathrm{kHz}$. By using the chopper technique, the input-referred noise of the chopper IA can be reduced to $0.28 \mu \mathrm{Vrms}(0 \sim 96 \mathrm{kHz})$, with a chopping frequency of $83.3 \mathrm{kHz}$. An automatic frequency tuning loop (ATL) is employed to adjust the corner frequency of the LPF dynamically so that the frequency ratio between the chopping frequency and the LPF corner frequency is 8.3, ensuring a noise reduction of $36.7 \mathrm{~dB}$.
\end{abstract}

Keywords: automatic frequency tuning loop; chopper technique; instrumentation amplifier; low-pass filter

\section{Introduction}

To meet a variety of healthcare demands, physiological signal acquisition can be performed by implantable, wearable, or portable monitoring systems [1,2]. Recently, systems that can monitor physiological signals, e.g., electroencephalography (EEG), electrocardiography (ECG), blood pressure and glucose, have been proposed and demonstrated [3-5]. Chopper IAs are widely adopted in these systems for their advantageous low noise, high input impedance, and high common mode rejection ratio (CMRR).

The chopper modulation operation is describeted as follows [6]: The bandwidth of the signal is assumed to be less than half the chopping frequency. The input chopper up-converts the signal to the chopping frequency and its odd harmonics, and then delivers it to the amplifier. After amplification, the signal is then down-converted to its original band by the output chopper. Because the DC offset and noise only go through the chopper modulation once, they are up-converted to the chopping frequency and its odd harmonics. The low-pass filter (LPF) then attenuates the up-converted noise. The amount of noise reduction depends on the ratio of the LPF corner frequency to the chopping frequency. However, both the corner frequency of the LPF and the chopping frequency may change due to process variations, which can diminish the amount of noise reduction.

In this work, strong correlation is built up between the corner frequency of the LPF and the chopping frequency so that the noise reduction is not affected by process variations. The rest of the paper is organized as follows. The system architecture and the way that the chopper IA cooperates 
with the automatic frequency tuning loop (ATL) to achieve the above-mentioned goal is introduced in Section 2. The design of the building blocks is addressed in Section 3. The measurement results are reported in Section 4. Finally, this work is summarized in Section 5.

\section{System Architecture}

A block diagram of the proposed chopper IA, which employs an ATL to control the corner frequency of the LPF for noise-reduction, is shown in Figure 1. CLK_chop is the chopping signal and CLK_ATL is the reference clock signal of the ATL. The former is generated from the configuration of an on-chip ring oscillator followed by a divide-by-12 circuit, while the latter is generated from the configuration of the same ring oscillator followed by a divide-by- 25 circuit. The chopping frequency is represented by f_chop, and the frequency of the ATL reference clock, by f_ATL. Note that the corner frequency of the LPF is equal to one fourth of $f_{-}$ATL. In this way, the ratio between the chopping frequency f_chop and the LPF corner frequency is always 8.3, even though the oscillating frequency of the ring oscillator changes with process variations. As a result, the LPF ensures an attenuation of $36.7 \mathrm{~dB}$ at the chopping frequency to provide adequate noise reduction in the chopper IA. In this work, an on-chip ring oscillator at $1 \mathrm{MHz}$ is implemented. Therefore, the chopping signal of $83.33 \mathrm{kHz}$ is delivered to the chopper IA, while the reference clock of $40 \mathrm{kHz}$ is delivered to the ATL. During the operation, the input of the LPF is first connected to the divide-by-four circuit to form the frequency tuning loop for corner frequency calibration. After the calibration is completed, the input of the LPF is connected to the chopper IA for noise reduction.

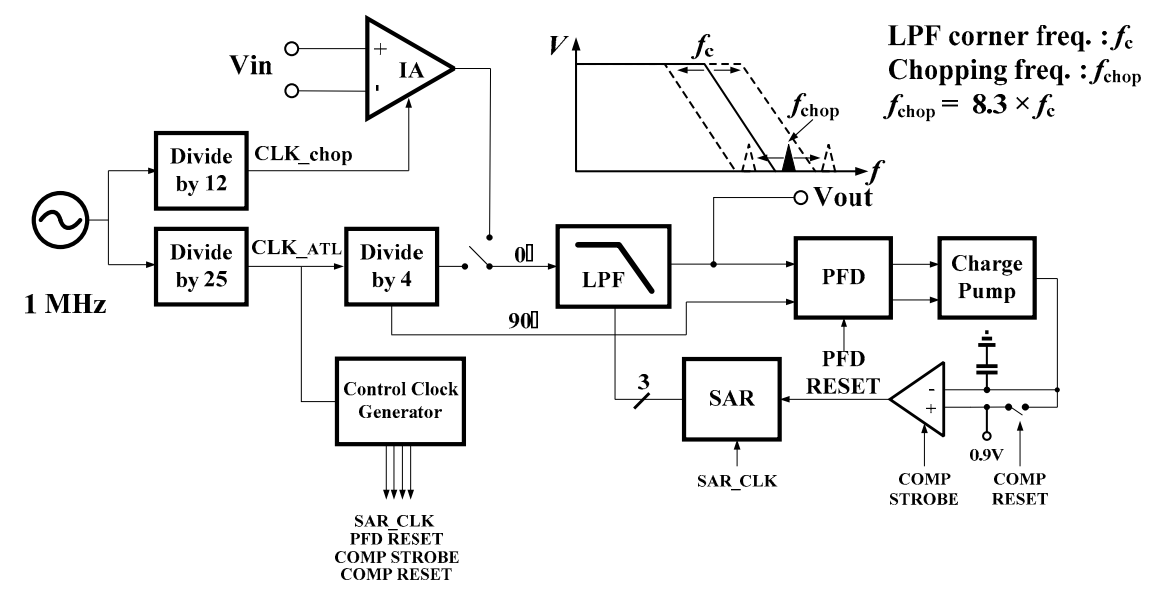

Figure 1. Block diagram of the proposed chopper IA with automatic frequency tuning loop (ATL).

In addition to the LPF, the ATL consists of a divide-by-four circuit, a phase frequency detector (PFD), a charge pump (CP), a comparator and a successive approximation register (SAR) [7,8]. The divide-by-four circuit generates a pair of quadrature signals at $10 \mathrm{kHz}$, namely, signals with $90^{\circ}$ phase difference. In Figure 1, the one that is sent to the LPF is defined as the $0^{\circ}$ signal and the other is defined as the $90^{\circ}$ signal. When the $0^{\circ}$ signal passes though the LPF, the filter introduces a frequency-dependent phase delay. Theoretically, the introduced phase delay should be $90^{\circ}$ at the corner frequency of the LPF. Therefore, the frequency tuning/calibration can be performed by measuring the introduced phase delay. There are three key steps in each tuning cycle. First, the output signal of the LPF, the $0^{\circ}$ signal with introduced phase delay, is compared with the $90^{\circ}$ signal in the PFD. Secondly, the PFD output signals modulate the charge/discharge currents of $\mathrm{CP}$, and the $\mathrm{CP}$ output is compared with a reference voltage of $0.9 \mathrm{~V}$ in the comparator to deliver a digital output. Then, the SAR logic sets one bit of the 3-bit control code of the LPF in accordance with this digital output signal. It takes three tuning cycles to complete the frequency tuning. Ideally, the Sallen-Key LPF would exhibit the corner frequency of $10 \mathrm{kHz}$ after the frequency tuning. 


\section{Circuit Design}

\subsection{Chopper Instrumentation Amplifier: Chopper IA}

The two-stage variable-gain chopper IA is developed from a difference amplifier, as depicted in Figure 2 [9]. The variable-gain function is realized by a switch-resistor array and a 3-to-8 decoder, where a unit resistor of $1.14 \mathrm{k} \Omega$ is adopted and the maximum resistance is $333 \mathrm{k} \Omega$. The chopper stabilization technique is performed by using two chopper-stabilized operational amplifiers, OP1 and $\mathrm{OP} 2$, in the first stage because the noise of the first stage is most critical [9].

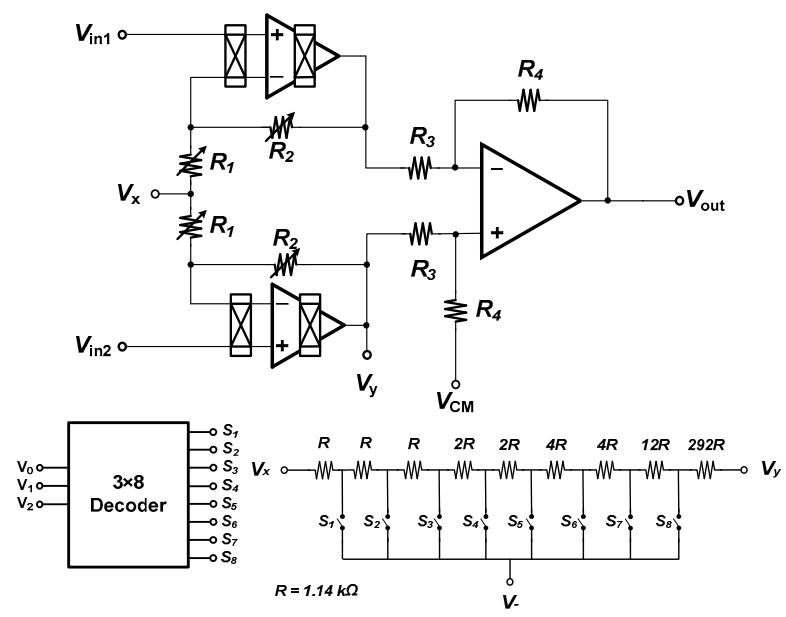

Figure 2. Tunable gain chopper IA [9].

\subsubsection{Chopper Operational Amplifier}

A schematic of the chopper-stabilized operational amplifier is shown in Figure 3 [9]. As previously mentioned, the noise performance of a two-stage amplifier is dominated in the first stage. If the noise origin of the operational amplifiers in the first stage can be removed, the noise performance of the IA can be significantly improved. The chopper modulator at the input, represented by chopper1, translates the input signal from its original band to the chopping frequency. The other two chopper modulators, chopper2 P-type and chopper2 N-type, then translate the desired signal back to its original band while converting the flicker noise, DC offsets, or noises well below the chopping frequency to the chopping frequency.

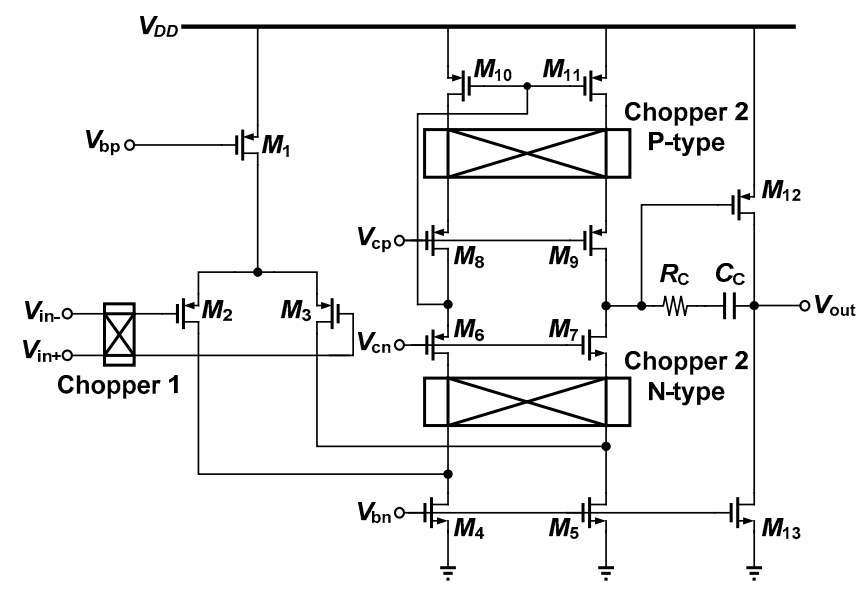

Figure 3. Chopper operational amplifier [9]. 


\subsubsection{Chopper Modulator}

The configuration of the chopper modulator and the switch implementation are shown in Figure $4 \mathrm{a}, \mathrm{b}$, respectively. To deal with imperfections such as the charge injection and clock feedthrough, dummies are added to the switches at both the sources and drains. When the switches are off, the channel charges are canceled by the dummies to prevent an electric potential error at the output. The switches are driven by non-overlapping clocks $\phi 1$ and $\phi 2$. During the operation, the input signals at nodes in 1 and in 2 are alternatively directed to either the node out 1 or out 2.

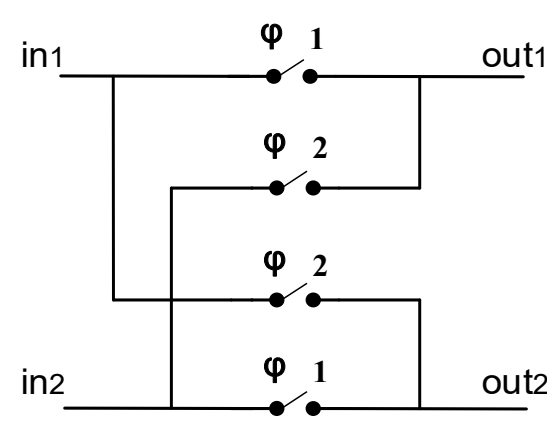

(a)

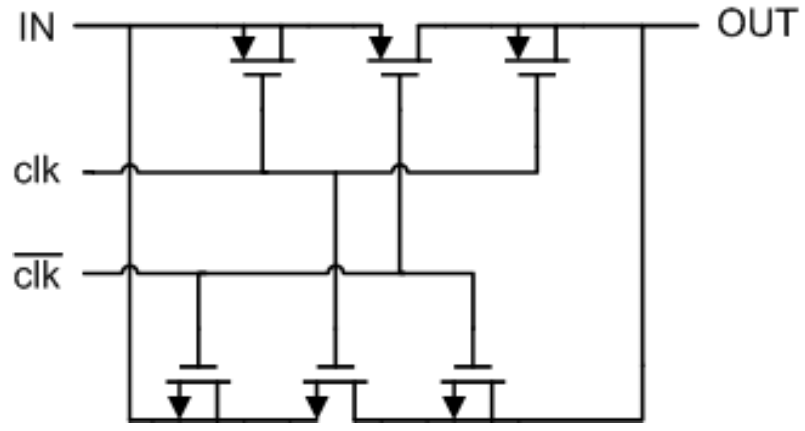

(b)

Figure 4. (a) Chopper modulation topology; (b) Switch topology.

The non-overlapping clock generator is shown in Figure 5a, and the waveform of the non-overlapping clock signals $\phi 1$ and $\phi 2$ is shown in Figure 5b. The non-overlapping clock generator is used to provide the required clocks $\phi 1$ and $\phi 2$ for the chopper modulator. The signal $\phi_{\text {chop }}$ is a square wave generated from the configuration of the on-chip ring oscillator followed by a divide-by-12 circuit, as previously mentioned. Notably, $\phi 1$ and $\phi 2$ need to be a pair of non-overlapping clock signals so that the switches driven by different clock signals are not turned on simultaneously. The inverting clock signals are also generated to control the dummy switches. The multi-inverter buffer stage is adopted to boost the driving capability of the clock signals.

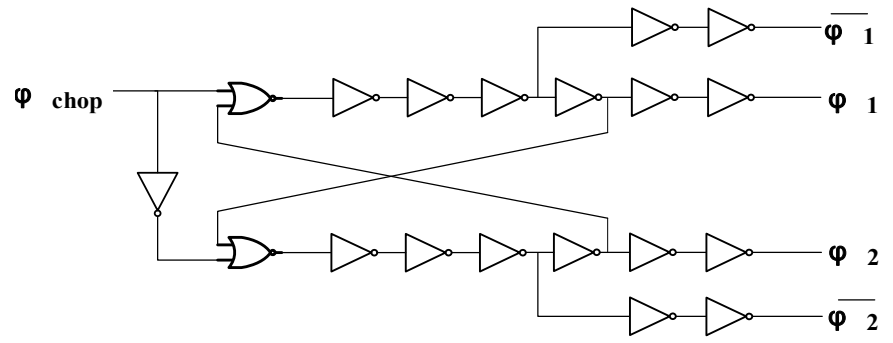

(a)

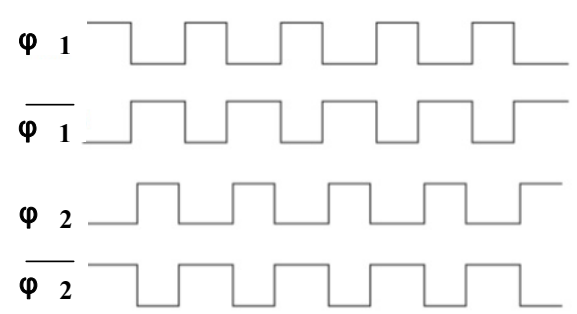

(b)

Figure 5. (a) Non-overlapping clock generator; (b) Non-overlapping clock signals.

The input-referred noise simulation results are obtained from the chopper IA before and after the chopper modulator are activated, as shown in Figure 6. The input-referred noise is greatly reduced after the chopper modulator is activated, with the chopping clock at $83.3 \mathrm{kHz}$. When the chopper modulator is activated, the input-referred noise at $100 \mathrm{~Hz}$ and $1 \mathrm{kHz}$ are $32.2 \mathrm{nV} / \sqrt{ } \mathrm{Hz}$ and $27.9 \mathrm{nV} / \sqrt{ } \mathrm{Hz}$, respectively. The transient simulation is also performed, as shown in Figure 7 . A $1 \mathrm{kHz}$ differential signal, with a DC level of $600 \mathrm{mV}$ and an amplitude of $0.5 \mathrm{mV}$, is applied at the input of the chopper IA. According to the simulation results, the output signals exhibit ignorable distortion, where the total harmonic distortions observed at the IA output node and LPF output node are $74.6 \mathrm{~dB}$ and $51.6 \mathrm{~dB}$, respectively. 


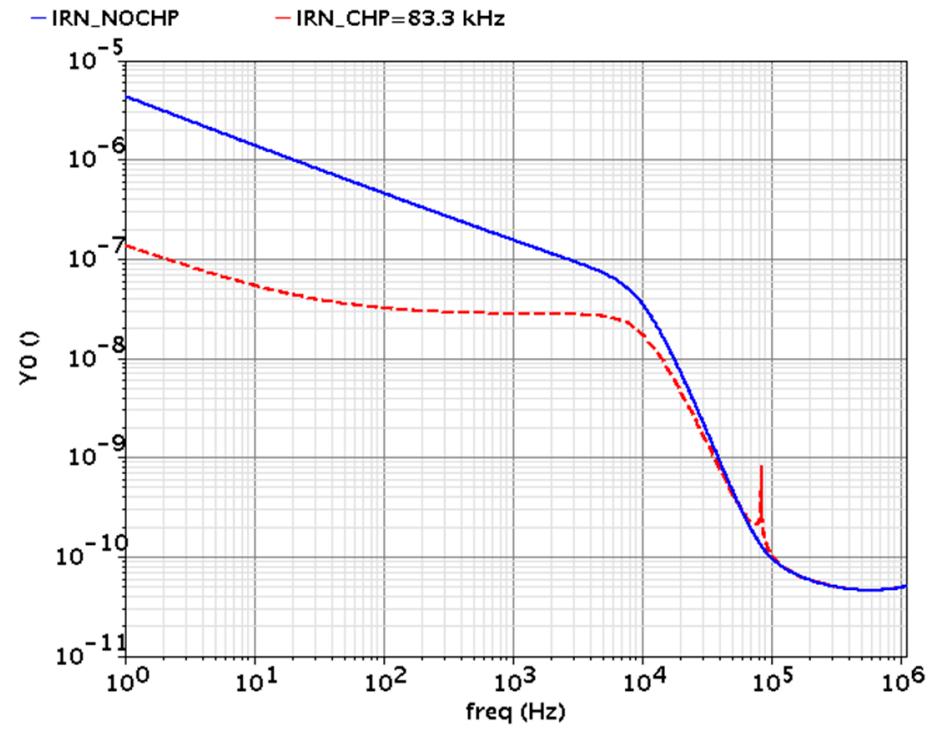

Figure 6. Input-referred noises of the chopper IA before (red dot line) and after (blue solid line) the chopper modulator is activated.

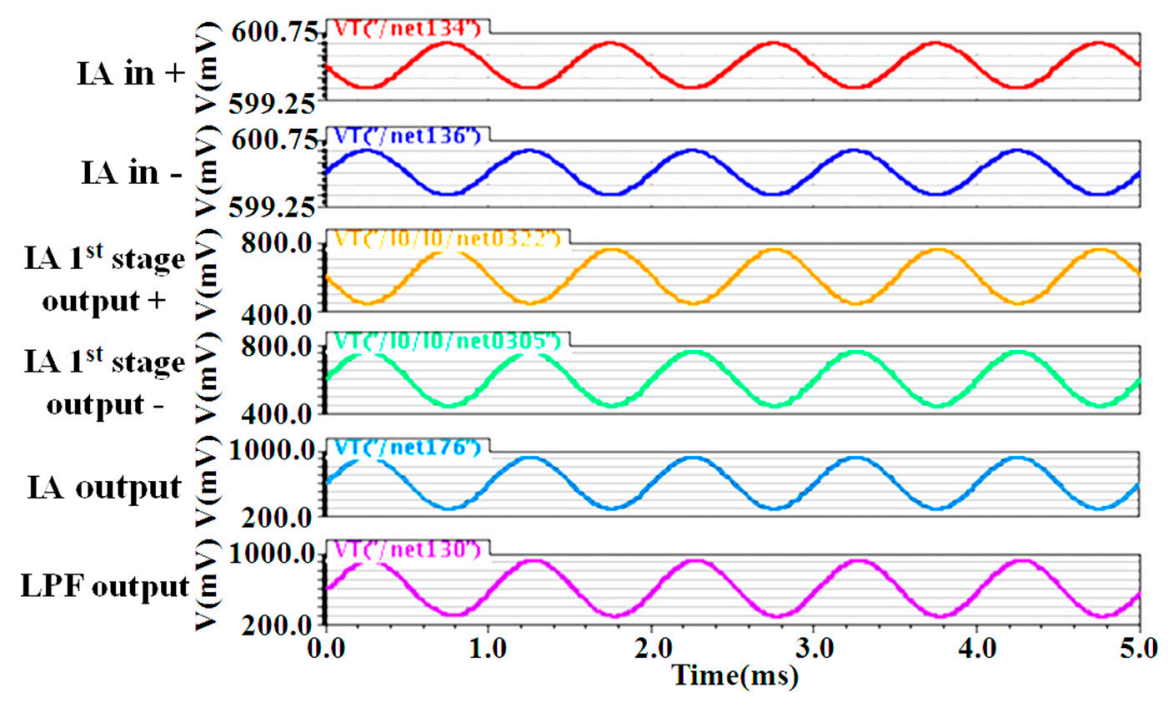

Figure 7. Transient simulation of the chopper IA.

\subsection{Sallen-Key Low Pass Filter}

As the last stage in the chopper IA, the LPF attenuates the up-converted noise at the chopping frequency. A schematic of the Sallen-Key LPF [10], where both variable capacitors C1 and C2 are realized by 3-bit cap-arrays and R1-2 = $1 \mathrm{M} \Omega$, is shown in Figure 8a. The schematic of the cap-array is shown in Figure 8b, where the capacitance of a unit capacitor is $104.0 \mathrm{fF}$. With a 3-bit binary-to-thermometer decoder, the LPF can be operated in 8 modes, where the capacitors $\mathrm{C} 1$ and $\mathrm{C} 2$ can be varied from $14.6 \mathrm{pF}$ to $29.7 \mathrm{pF}$ and $7.3 \mathrm{pF}$ to $14.9 \mathrm{pF}$, respectively. The frequency response simulation results of the LPF are shown in Figure 9, where 8 different corner frequencies are realized. According to the simulation results, the corner frequency of the LPF can be varied from $6.9 \mathrm{kHz}$ to $13.6 \mathrm{kHz}$ with a step size of $0.8 \sim 1 \mathrm{kHz}$. 


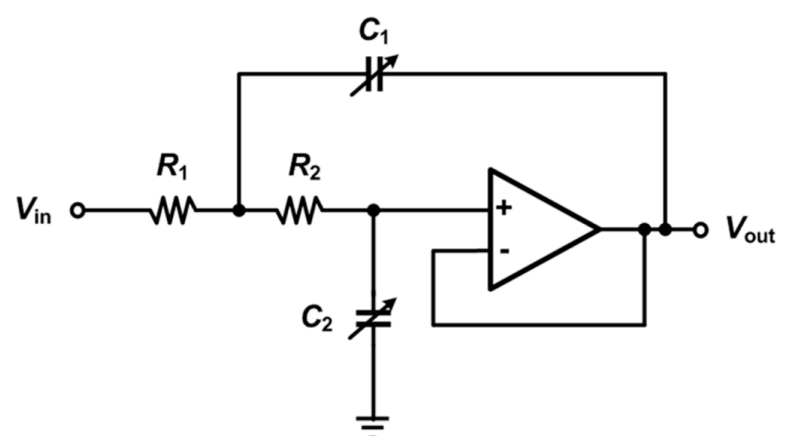

(a)
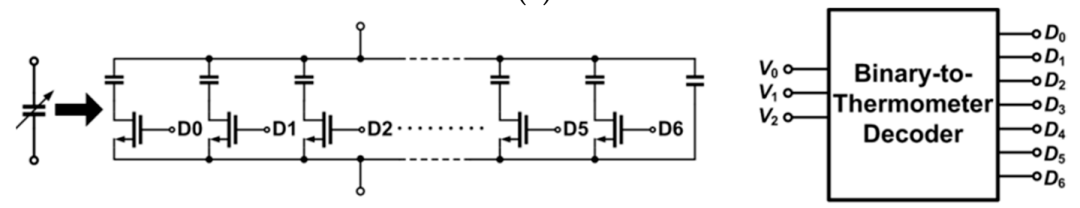

(b)

Figure 8. (a) Sallen-Key low-pass filter; (b) Cap-array schematic.

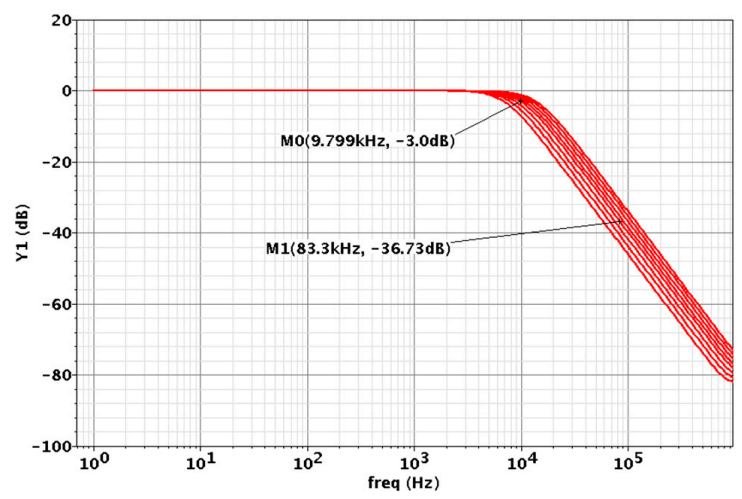

Figure 9. Frequency response simulation results of the low pass filter (LPF).

\subsection{Divide-by-Four Circuit}

The divide-by-four circuit is used to generate the required quadrate signals. The D flip-flop topology is shown in Figure 10a. The nodes " $\mathrm{D}$ " and "QN" are connected to form a divide-by-two circuit. The divide-by-four circuit is realized by two divide-by-two circuits in cascade, as shown in Figure 10b. The input clock and output signal waveforms obtained from the simulation of the divide-by-four circuit are shown in Figure 10c. The $0^{\circ}$ signal (OUT_0) and the $180^{\circ}$ signal (OUT_180) are differential signals that are fed to the LPF, while the $90^{\circ}$ signal (OUT_90) is sent to the PFD.

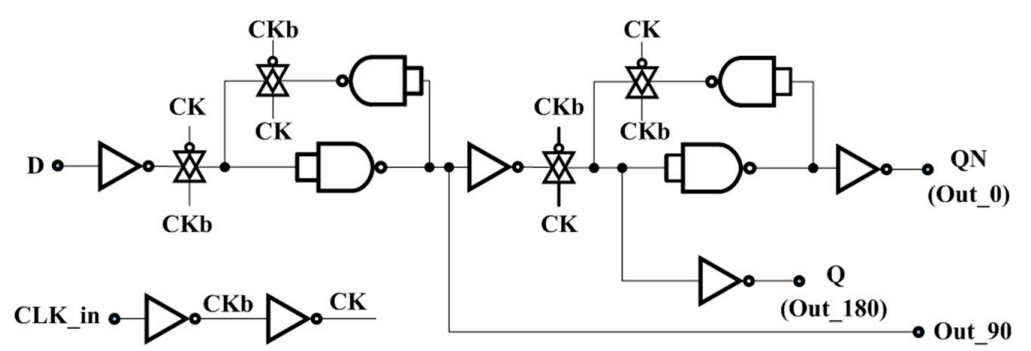

(a)

Figure 10. Cont. 


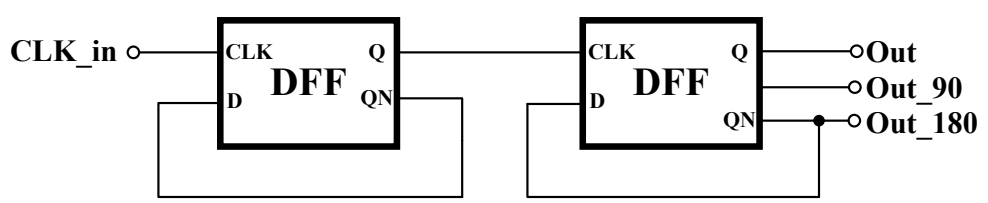

(b)

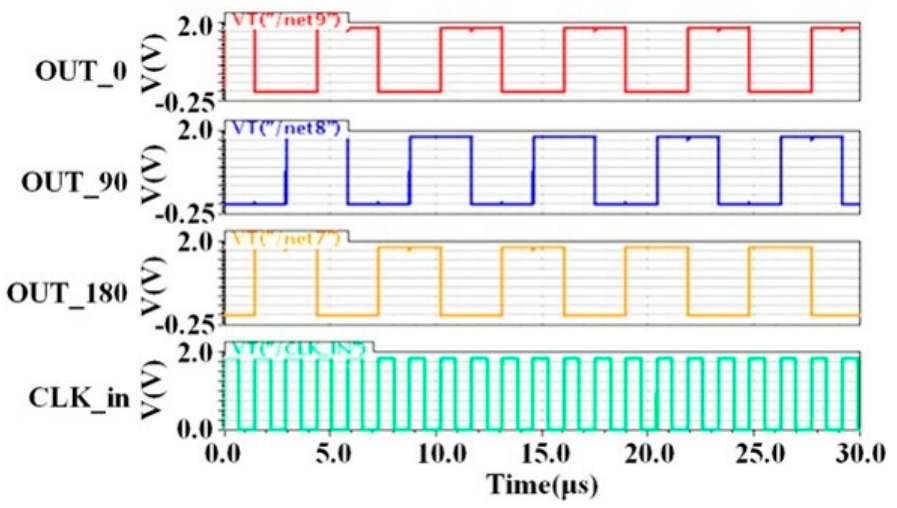

(c)

Figure 10. (a) D-flip-flop logic diagram; (b) Divide-by-four block diagram; (c) Divide-by-four simulation results.

\subsection{Control Clock Generator}

The required timing control signals are generated by a control clock generator that is designed with Verilog code and then realized through logic synthesis. The transient waveforms of the input clock and the four timing control clocks, generated from the control clock generator, are shown in Figure 11. The timing control of the ATL is performed as follows. The comparator would be activated by the "Comp strobe", which is negative-edge triggered. The result of the comparison is then sent to the SAR logic. At the same time, the PFD is deactivated by the "PFD reset", which is positive-edge triggered. Then, the SAR logic is activated by "SAR CLK" to determine one bit of the binary codes according to the comparison result. Finally, the comparator is deactivated by the "Comp reset", which is positive-edge triggered.

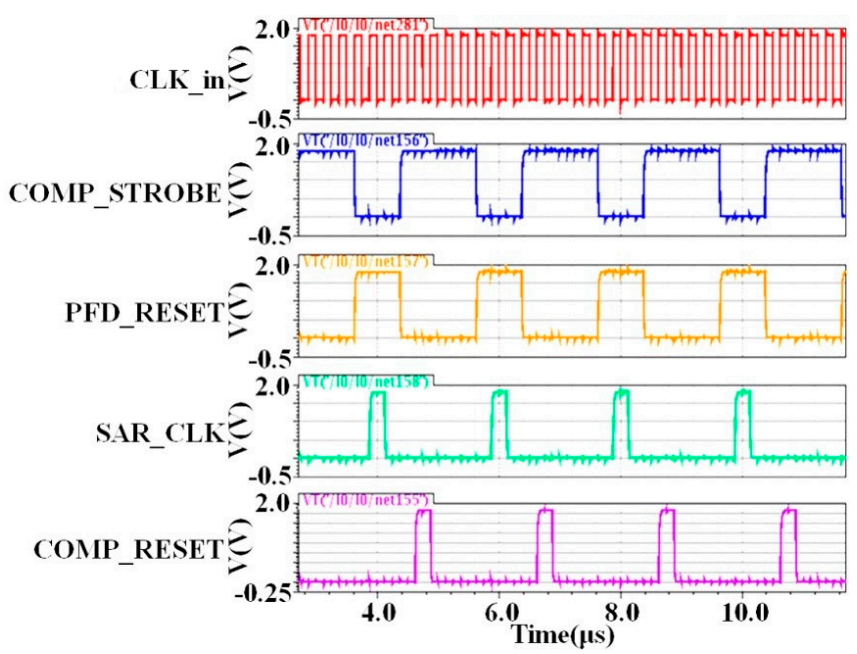

Figure 11. Simulation of the timing control. 


\subsection{Automatic Frequency Tuning Loop (ATL)}

When the ATL is applied with different input clocks, the 3-bit digital output of the SAR logic can be observed to verify the frequency tuning function. The digital code is 111 when an input clock of $24.8 \mathrm{kHz}$ is applied to the ATL, as shown in Figure 12a. The digital code is 011 when an input clock of $42.8 \mathrm{kHz}$ is applied to the ATL, as shown in Figure $12 \mathrm{~b}$. The digital code is 000 when an input clock of $60 \mathrm{kHz}$ is applied to the ATL, as shown in Figure 12c. Note that the 3-bit digital code controls the variable capacitors in the LPF and determines the corner frequency of the LPF. Therefore, according to the simulation results, the corner frequency of the LPF can be adjusted by the input clock.

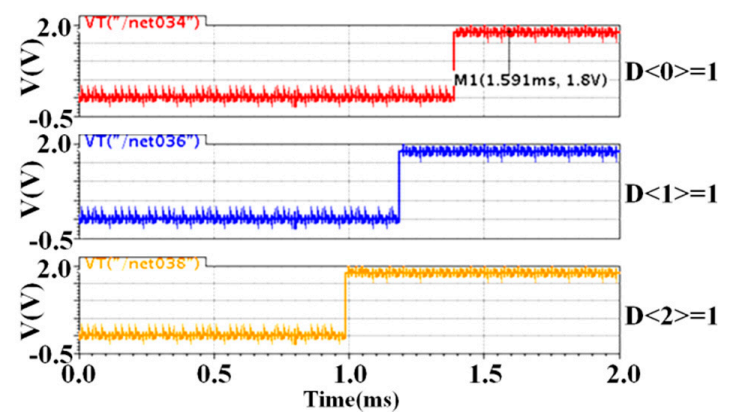

(a)

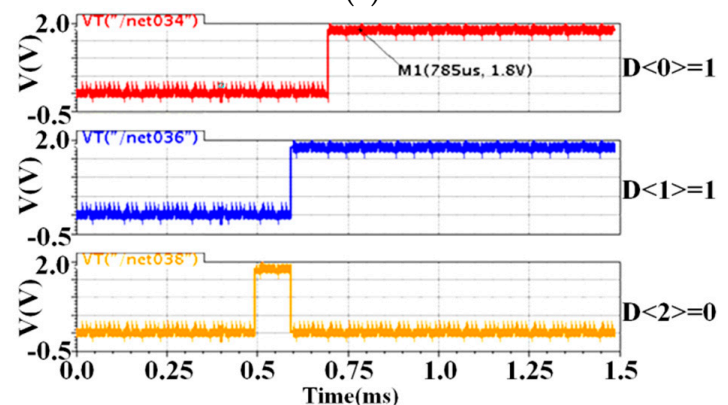

(b)

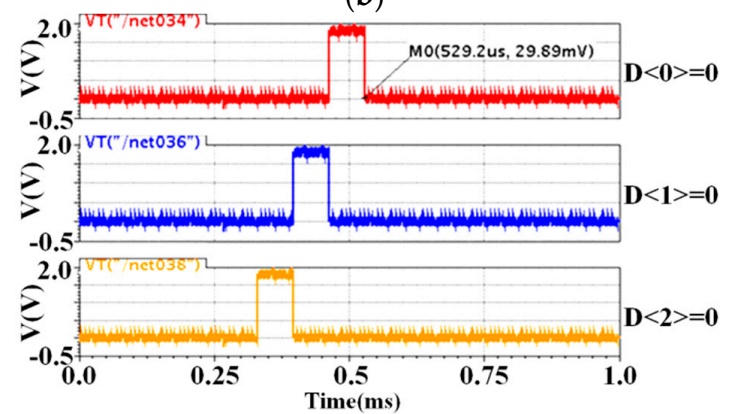

(c)

Figure 12. The 3-bit digital code observed in the ATL simulation with an input clock of (a) $24.8 \mathrm{kHz}$, (b) $42.8 \mathrm{kHz}$ and (c) $60.0 \mathrm{kHz}$.

\section{Measurement}

\subsection{Chopper IA}

The measurement of the chopper IA is performed with the Anritsu network analyzer MS4630B. The measured frequency response of the chopper IA is shown in Figure 13, where the gain of the chopper IA can be varied from $20.7 \mathrm{~dB}$ to $48.5 \mathrm{~dB}$ and the corner frequency is $6.7 \sim 7.7 \mathrm{kHz}$. The measured CMRR of the chopper IA is shown in Figure 14. The CMRR is above $95 \mathrm{~dB}$ in the frequency range below $10 \mathrm{kHz}$. The noise measurement is performed using the R\&S UPV audio analyzer (Rohde \& Schwarz, Munich, Germany). During the measurement, the ATL is activated to perform 
the frequency tuning, where an input clock of $40 \mathrm{kHz}$ is provided by the configuration of the 1-MHz on-chip ring oscillator followed by a divide-by- 25 circuit, so that the corner frequency of the LPF is set to $10.1 \mathrm{kHz}$. Three chip samples have been tested. The average of the input-referred noises obtained from these chips, before and after the chopper modulator is activated, is shown in Figure 15. The in-band noise is lowered by $40 \mathrm{~dB}$ after the chopper modulator is activated. When the chopper IA operates in the highest-gain mode, the input-referred noise, integrated from 0 to $96 \mathrm{kHz}$, is $0.28 \mathrm{uVrms}$.

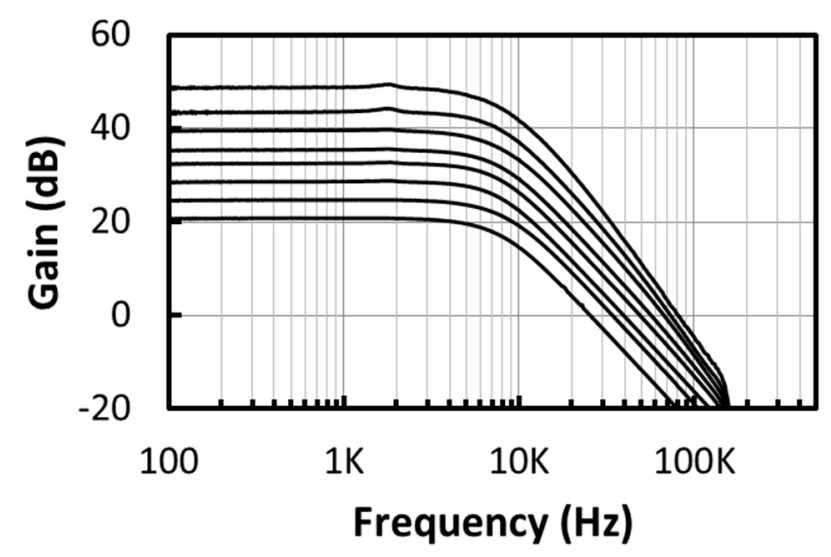

Figure 13. The measured frequency responses of the variable-gain chopper IA.

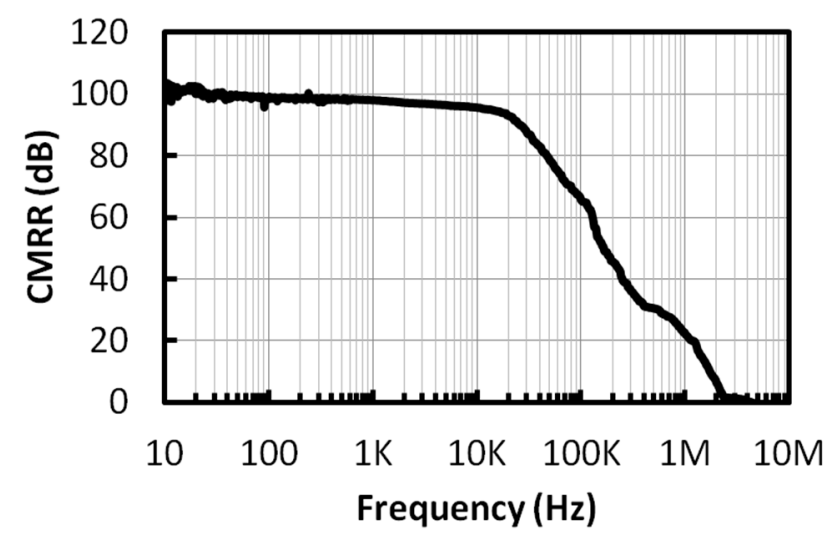

Figure 14. The measured CMRR of the chopper IA.

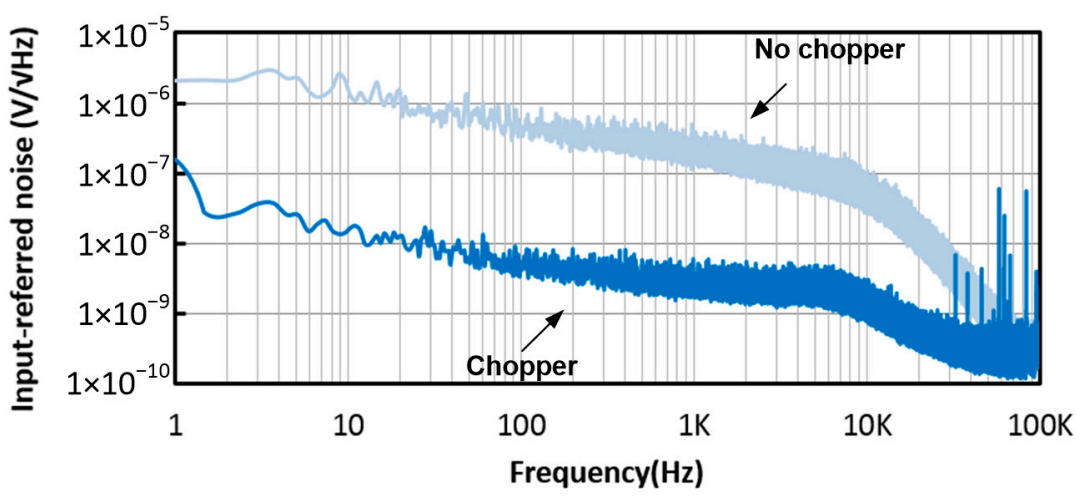

Figure 15. The measured input-referred noise of the chopper IA before and after the chopper modulator is activated. 


\section{2. $L P F$ and $A T L$}

The measurement of the LPF is performed with the Anritsu network analyzer MS4630B (Anritsu Company Inc., Kanagawa Prefecture, Japan). The measured gain and phase frequency responses of the LPF are shown in Figure 16. The corner frequency of the LPF can be varied from $6.7 \mathrm{kHz}$ to $12.8 \mathrm{kHz}$ with a step size of $0.9-1.0 \mathrm{kHz}$. Note that the frequency at which the phase delay reaches $90^{\circ}$ is from $7.5 \mathrm{kHz}$ to $14.4 \mathrm{kHz}$.

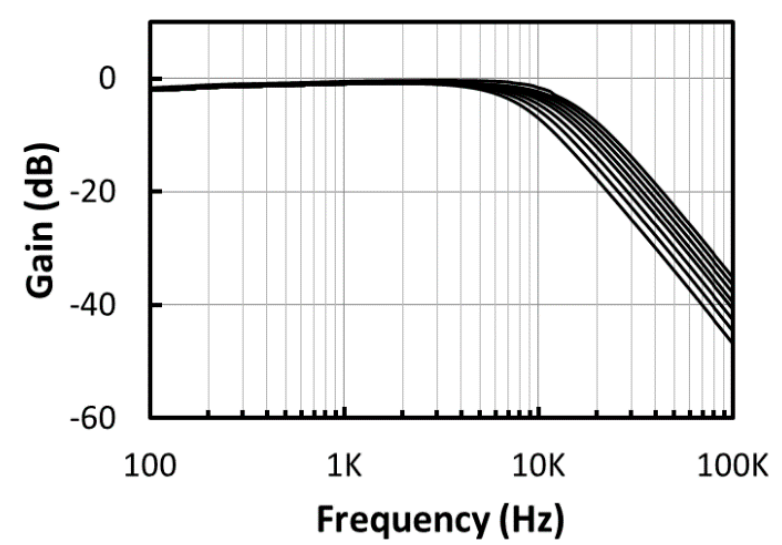

(a)

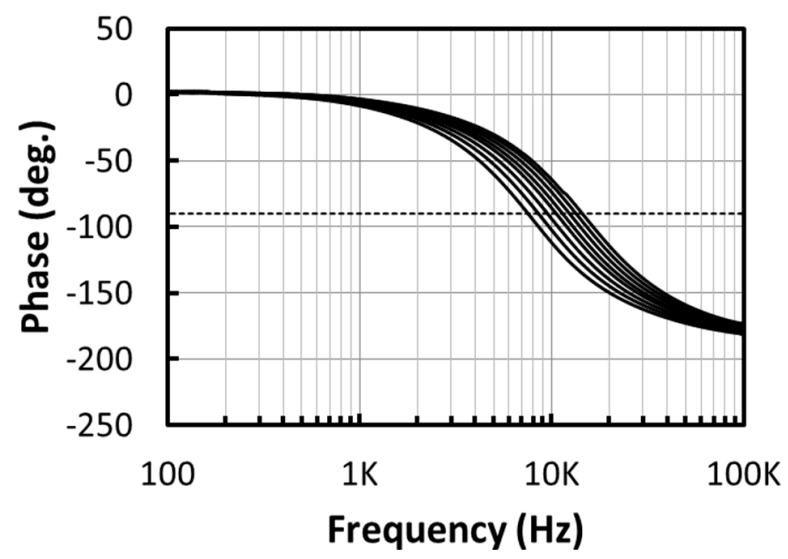

(b)

Figure 16. The measured (a) gain and (b) phase frequency responses of the LPF.

The function of the ATL was verified by observing the digital output of the ATL under different input clocks. During the measurement, the 3-bit digital output of the SAR logic was observed with a logic analyzer, where the input clock was provided by a function generator. The measurement results of digital output under input clocks from $32.8 \mathrm{kHz}$ to $58.4 \mathrm{kHz}$ are listed in Table 1.

Table 1. Digital Codes under Different Input Clocks.

\begin{tabular}{cc}
\hline CLK/4 (kHz) & Digital Codes \\
\hline$>14.6$ & 000 \\
$13.7 \sim 14.5$ & 001 \\
$12.5 \sim 13.6$ & 010 \\
$11.4 \sim 12.4$ & 011 \\
$10.4 \sim 11.3$ & 100 \\
$9.2 \sim 10.3$ & 101 \\
$8.3 \sim 9.1$ & 110 \\
$<8.2$ & 111 \\
\hline
\end{tabular}


The digital outputs of the SAR logic, observed with the logic analyzer under three different clocks, are shown in Figure 17. When an input clock of $24.8 \mathrm{kHz}$ is applied, the observed digital code is 111 after frequency tuning. When an input clock of $42.8 \mathrm{kHz}$ is applied, the observed digital code is 100 after frequency tuning. When an input clock of $60.0 \mathrm{kHz}$ is applied, the observed digital code is 000 after frequency tuning. According to the measurement results, the corner frequency of the LPF can be controlled by the input clock. The frequency response of the LPF for an ATL input clock of $28 \mathrm{kHz}$, $42.8 \mathrm{kHz}$ and $60 \mathrm{kHz}$, where the corner frequency of the LPF is adjusted to $6.7 \mathrm{kHz}, 10.1 \mathrm{kHz}$ and $12.8 \mathrm{kHz}$, respectively, is shown in Figure 18.

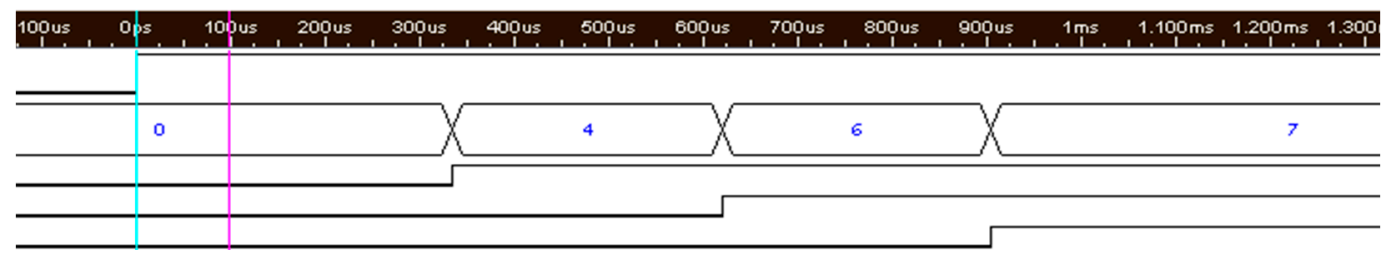

(a)

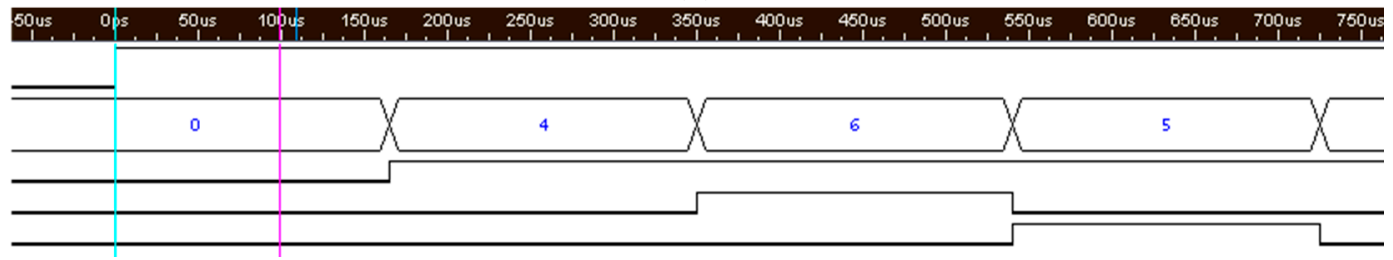

(b)

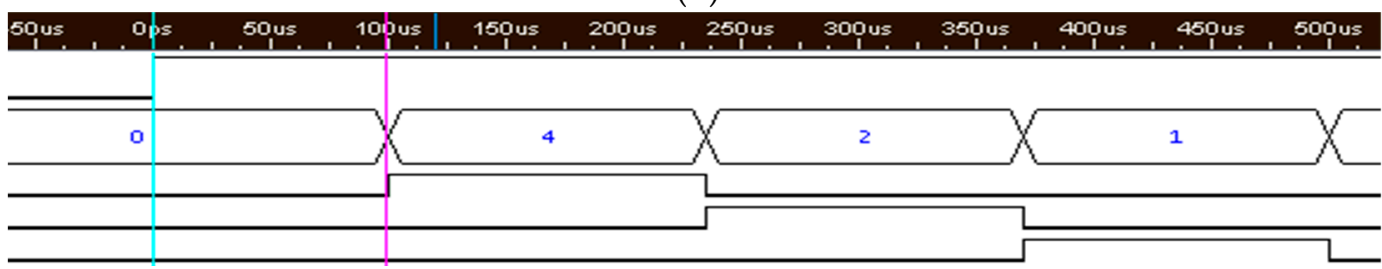

(c)

Figure 17. The measurement results of ATL: (a) Input clock of $24.8 \mathrm{kHz}$; ATL digital outputs = 111; (b) Input clock of $42.8 \mathrm{kHz}$; ATL digital outputs =100; (c) Input clock of $60.0 \mathrm{kHz}$; ATL digital outputs $=000$.

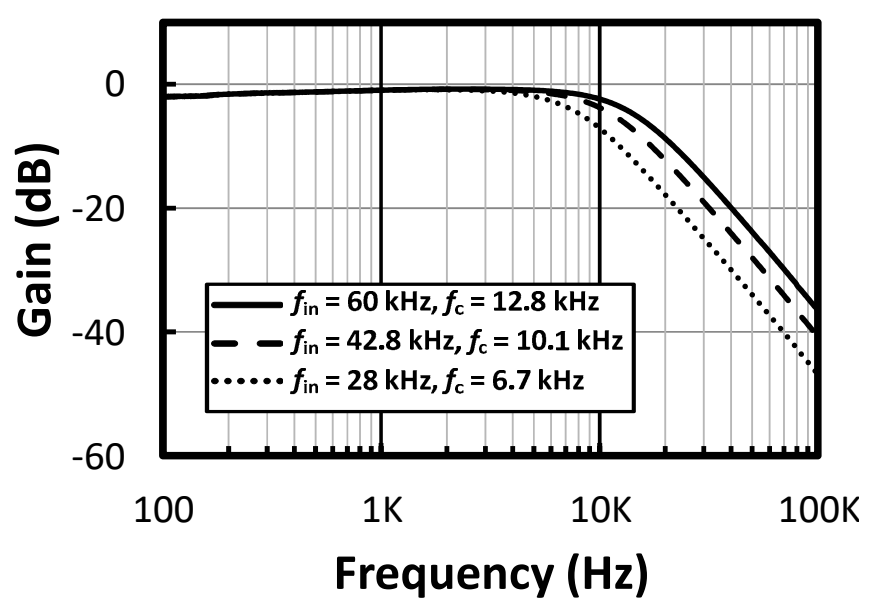

Figure 18. The measured frequency responses of LPF for an ATL input clock of 28, 42.8 and $60 \mathrm{kHz}$.

The chopper IA was fabricated with TSMC $0.18 \mu \mathrm{m}$ CMOS technology. The chip micrograph, where the chip occupies an area of $2.13 \mathrm{~mm}^{2}$, is shown in Figure 19. The performance of the chopper IA, along with other previous works for comparison [11-13], is summarized in Table 2. 


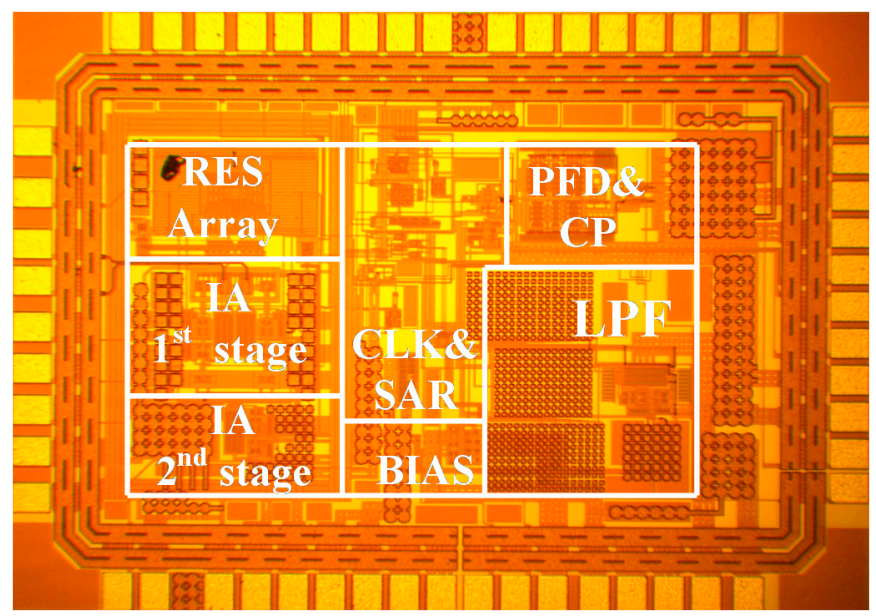

Figure 19. Chip micrograph of the chopper IA with an ATL.

Table 2. Performance Summary of the Chopper IA.

\begin{tabular}{|c|c|c|c|c|}
\hline Parameter (Unit) & This Work & [11] & [12] & [13] \\
\hline Technology (nm) & 180 & 65 & 180 & 65 \\
\hline Supply Voltage (V) & 1.2 & 1.2 & 0.5 & 1 \\
\hline Chopping Frequency (kHz) & 83.3 & 50 & 5 & 5 \\
\hline Gain $(\mathrm{dB})$ & $20.7 \sim 48.5$ & 34 & 39.6 & 100 \\
\hline $\mathrm{f} 3 \mathrm{~dB}(\mathrm{kHz})$ & $6.7 \sim 7.7$ & 11 & 0.25 & 0.7 \\
\hline Common Mode Rejection Ratio (dB) & $>95$ & $>94$ & $>106$ & 134 \\
\hline Power Supply Rejection Ratio (dB) & $>95$ & $>100$ & $>73$ & 120 \\
\hline Input-Referred Noise $(\mathrm{nV} / \sqrt{ } \mathrm{Hz})$ & 4.2 & 37 & 112 & 60 \\
\hline Input-Referred Noise (uVrms) & $\begin{array}{l}0.205(0.5-100 \mathrm{~Hz}) \\
0.213(0.5-250 \mathrm{~Hz}) \\
0.345(0.0-96 \mathrm{kHz})\end{array}$ & $\mathrm{N} / \mathrm{A}$ & $2.8(0.5-250 \mathrm{~Hz})$ & $6.7(0.5-100 \mathrm{~Hz})$ \\
\hline Noise Efficiency Factor [14] & 6.13 & 2.0 & 8.7 & 3.9 \\
\hline Power $(\mathrm{mW})$ & 1.1 & 0.0014 & 0.0013 & 0.0021 \\
\hline Chip Size $\left(\mathrm{mm}^{2}\right)$ & 2.13 & $\mathrm{~N} / \mathrm{A}$ & 1 & 0.2 \\
\hline
\end{tabular}

\section{Conclusions}

A low-noise IA is designed and implemented by using the chopper technique, where an LPF is employed to attenuate the up-converted noise at chopping frequency. With an ATL, the corner frequency of the LPF can be controlled with the input clock. The on-chip ring oscillator and dividers are employed to generate the input clock of the ATL and the clock of the chopper modulator, so that the noise reduction can be immune to process variations as the two clocks are correlated. During the measurement, the corner frequency of the LPF is set to $10.1 \mathrm{kHz}$, while the chopper modulator operates at a clock of $83.3 \mathrm{kHz}$. The chopper IA consumes $1.1 \mathrm{~mW}$ from a supply voltage of $1.2 \mathrm{~V}$. The chopper IA delivers a variable gain from $20.7 \mathrm{~dB}$ to $48.5 \mathrm{~dB}$ and achieves a minimum bandwidth of $6.7 \mathrm{kHz}$. The input-referred noise of $0.28 \mu \mathrm{Vrms}(0 \sim 96 \mathrm{kHz})$ is achieved.

Author Contributions: H.-C.C. conceived this work; C.-M.W., M.-Y.Y., and S.-C.Y. designed the circuits and implemented the chip; C.-M.W. wrote the major draft; H.-C.C. assisted with review of the paper.

Acknowledgments: This work was supported in part by MediaTek and the Ministry of Science and Technology, Taiwan, under the contract number MOST 105-2622-8-002-002, and in part by the Ministry of Science and Technology, Taiwan, under the contract number MOST 106-2221-E-011-158. We are grateful to the National Chip Implementation Center of Taiwan for their technical support during the chip fabrication.

Conflicts of Interest: The authors declare no conflict of interest. 


\section{References}

1. Yazicioglu, R.F.; Kim, S.; Torfs, T.; Kim, H.; van Hoof, C. A $30 \mu \mathrm{W}$ Analog signal processor ASIC for portable biopotential signal monitoring. IEEE J. Solid-State Circuits 2011, 46, 209-223. [CrossRef]

2. Gyselinckx, B. Human++: Emerging Technology for Body Area Networks. In Proceedings of the 2006 IFIP International Conference on Very Large Scale Integration, Nice, France, 16-18 October 2006; pp. 175-180.

3. Moy, T.; Huang, L.; Rieutort-Louis, W.; Wu, C.; Cuff, P.; Wagner, S.; Sturm, J.C.; Verma, N. An EEG acquisition and biomarker-extraction system using low-noise-amplifier and compressive-sensing circuits based on flexible, thin-film electronics. IEEE J. Solid-State Circuits 2017, 52, 309-321. [CrossRef]

4. Mohan, R.; Zaliasl, S.; Gielen, G.G.E.; van Hoof, C.; Yazicioglu, R.F.; van Helleputte, N. A 0.6-V, 0.015-mm², time-based ECG readout for ambulatory applications in 40-nm CMOS. IEEE J. Solid-State Circuits 2017, 52, 298-308. [CrossRef]

5. Song, K.; Ha, U.; Park, S.; Bae, J.; Yoo, H.J. An impedance and multi-wavelength near-infrared spectroscopy IC for non-invasive blood glucose estimation. IEEE J. Solid-State Circuits 2015, 50, 1025-1037. [CrossRef]

6. Bakker, A.; Thiele, K.; Huijsing, J.H. A CMOS nested-chopper instrumentation amplifier with 100-nV offset. IEEE J. Solid-State Circuits 2000, 35, 1877-1883. [CrossRef]

7. Rogin, J.; Kouchev, I.; Brenna, G.; Tschopp, D.; Huang, Q. A 1.5-V 45-mW direct-conversion WCDMA receiver IC in 0.13- $\mu \mathrm{m}$ CMOS. IEEE J. Solid-State Circuits 2003, 38, 2239-2248. [CrossRef]

8. Chen, H.C.; Wang, T.; Chiu, H.W.; Yang, Y.C.; Kao, T.H.; Huang, G.W.; Lu, S.S. A 5-GHz-band CMOS receiver with low LO self-mixing front end. IEEE Trans. Circuits Syst. I Regul. Pap. 2009, 56, 705-713. [CrossRef]

9. Lyu, Y.J.; Wu, Q.X.; Huang, P.S.; Chen, H.C. CMOS analog front end for ECG measurement system. In Proceedings of the 2012 International Symposium on Intelligent Signal Processing and Communications Systems, Taipei, Taiwan, 4-7 November 2012; pp. 327-332.

10. Kuo, P.Y.; Sie, L.F. Analyze the behavior model based on Verilog-A for Sallen-Key low-pass filter. In Proceedings of the 2015 IEEE International Conference on Consumer Electronics-Taiwan, Taipei, Taiwan, 6-8 June 2015; pp. 460-461.

11. Chandrakumar, H.; Marković, D. A simple area-efficient ripple-rejection technique for chopped biosignal amplifiers. IEEE Trans. Circuits Syst. II Express Briefs 2015, 62, 189-193. [CrossRef]

12. Zhu, Z.; Bai, W. A 0.5-V 1.3- $\mathrm{WW}$ analog front-end CMOS circuit. IEEE Trans. Circuits Syst. II Express Br. 2016, 63, 523-527. [CrossRef]

13. Fan, Q.; Sebastiano, F.; Huijsing, J.H.; Makinwa, K.A.A. A $1.8 \mu \mathrm{W} 60 \mathrm{nV} / \sqrt{ } \mathrm{Hz}$ Capacitively-coupled chopper instrumentation amplifier in $65 \mathrm{~nm}$ CMOS for wireless sensor nodes. IEEE J. Solid-State Circuits 2011, 46, 1534-1543. [CrossRef]

14. Zheng, J.; Ki, W.H.; Hu, L.; Tsui, C.Y. Chopper capacitively coupled instrumentation amplifier capable of handling large electrode offset for biopotential recordings. IEEE Trans. Circuits Syst. II Express Briefs 2017, 64, 1392-1396. [CrossRef]

(C) 2018 by the authors. Licensee MDPI, Basel, Switzerland. This article is an open access article distributed under the terms and conditions of the Creative Commons Attribution (CC BY) license (http://creativecommons.org/licenses/by/4.0/). 